\title{
Prognostic Predictability of American Joint Committee on Cancer 8th Staging System for Perihilar Cholangiocarcinoma: Limited Improvement Compared with the 7th Staging System
}

\author{
Jong Woo Lee, MD \\ Jae Hoon Lee, MD, PhD \\ Yejong Park, MD \\ Woohyung Lee, MD \\ Jaewoo Kwon, MD \\ Ki Byung Song, MD, PhD \\ Dae Wook Hwang, MD, PhD \\ Song Cheol Kim, MD, PhD
}

Department of Hepatobiliary and Pancreatic Surgery, Asan Medical Center, University of Ulsan College of Medicine, Seoul, Korea

\begin{abstract}
Purpose
This study was conducted to evaluate the prognostic values of the 7th and 8th American Joint Committee on Cancer (AJCC) staging systems for patients with resected perihilar cholangiocarcinoma (PHCC).
\end{abstract}

\section{Materials and Methods}

A total of 348 patients who underwent major hepatectomy for PHCC between 2008 and 2015 were identified from a single center. Overall survival (OS) was estimated using the Kaplan-Meier method and compared across stage groups with the log-rank test. The concordance index was used to evaluate the prognostic predictability of the 8th AJCC staging system compared with that of the 7th.

\section{Results}

In the 8th edition, the stratification of each group of T classification improved compared to that in the 7 th, as the survival rate of T4 decreased (T2, 31.2\%; T3, 13.9\%; T4, 15.1\%; T1T2, $p=0.260$; T2-T3, $p=0.001 ;$ T3-T4, $p=0.996)$. Both editions showed significant survival differences between each N category, except between N1 and N2 ( $p=0.063)$ in 7th edition. Differences of point estimates between the 8th and 7 th $\mathrm{T}$ and $\mathrm{N}$ classification and overall stages were $+0.028,+0.006$, and +0.039 , respectively $(T, p=0.005 ; N, p=0.115$; overall stage, $p=0.005$ ). In multivariable analysis, posthepatectomy liver failure, $\mathrm{T}$ category, $\mathrm{N}$ category, distant metastasis, histologic differentiation, intraoperative transfusion, and resection margin status were associated with OS.

\section{Conclusion}

The prognostic predictability of 8th AJCC staging for PHCC improved slightly, with statistical significance, compared to the 7 th edition, but its overall performance is still unsatisfactory.
Department of Hepatobiliary and Pancreatic Surgery, Asan Medical Center, University of Ulsan College of Medicine, 88 Olympic-ro 43gil, Songpa-gu, Seoul 05505, Korea

Tel: 82-2-3010-6730

Fax: 82-2-2045-4232

E-mail: hbpsurgeon@gmail.com

Received January 9,2020

Accepted March 11, 2020

Published Online March 12, 2020
Key words

Perihilar cholangiocarcinoma, Stage, Survival, Prognosis

\section{Introduction}

Cholangiocarcinoma can be classified into intrahepatic, perihilar, and distal bile duct cancer. Perihilar cholangiocarcinoma (PHCC) is relatively rare but one of the most difficult cancers to achieve curative resection in; thus, patients have a dismal prognosis even after surgery $[1,2]$.

A standardized staging system is crucial to determine an appropriate treatment plan. The tumor, node, metastasis
(TNM) system by the American Joint Committee on Cancer (AJCC) is used widely for this purpose. Prior to the 7th edition of the AJCC system, cholangiocarcinoma was classified as intrahepatic and extrahepatic; therefore, PHCC was classified as extrahepatic. In the 7th edition, PHCC was separated from distal cholangiocarcinoma.

Changes in the 8th AJCC staging system, summarized in Table 1 [3], include the following: In terms of the T category, the definition of Tis has been expanded to include high-grade biliary intraepithelial neoplasia (BillN-3). Bilateral second- 
order biliary radical invasion (Bismuth-Corlette type IV) has been removed from the T4 category. The $\mathrm{N}$ category has been reclassified, based on the number of positive nodes, to N1 (one to three positive nodes) and N2 (four or more positive nodes), whereas in the 7th AJCC edition, they were classified to N1 (regional lymph node [LN] metastasis) and N2 (paraaortic, aortocaval, superior mesenteric artery, and/or celiac artery LN metastasis). Regarding stage groups, T4 tumors have been downstaged from stage IVA to stage IIIB, since they are considered resectable with combined vascular resection and reconstruction. In addition, N1 has been changed from stage IIIB to IIIC, and N2 is classified as stage IVA.

The objective of this study was to evaluate the prognostic value of the 8th edition of the AJCC staging system in patients with resected $\mathrm{PHCC}$ by comparing it with the 7 th edition.

\section{Materials and Methods}

\section{Patient selection}

Between 2008 and 2015, 485 patients who underwent surgery for PHCC at Asan Medical Center were identified. Among them, patients who underwent bile duct resection only, those who had an unresectable tumor due to extensive local invasion or metastasis, and those whose final pathology identified a type other than PHCC were excluded.

\section{Definition}

PHCC was defined as cholangiocarcinoma involving the hilar bile duct (the duct located topologically between the right side of the umbilical portion of the left portal vein and the left side of the origin of the right posterior portal vein [2]). TNM stage was determined according to both the 7 th and 8th editions of the AJCC system. In pathological examination, resection margin was classified as microscopically negative (R0) and microscopically positive (R1). R0 was defined as no malignant tumor cells, biliary intraepithelial neoplasia (BilIN) 1, 2, and 3. Overall survival (OS) was defined as the period between the date of surgery and the date of death.

\section{Patient management}

Patients underwent major hepatectomy (resection of three segments or more) with en-bloc resection of the caudate lobe and extrahepatic bile duct according to the Bismuth classification, and the extent was evaluated with preoperative imaging computed tomography and magnetic resonance cholangiopancreatography [1]. All the soft tissue in the hepatoduodenal ligament were removed. LNs along the common hepatic artery (\#8), hepatoduodenal ligament (\#12), and posterior pancreaticoduodenal node (\#13) were routinely resected. Combined vascular resection and reconstruction was performed if the tumor involved the portal vein or hepatic artery to remnant side of liver.

\section{Staging}

Biliary extent of the tumor and Bismuth type were evaluated to determine T4 category in the 7th edition of the AJCC system. We determined T3 and T4 in both editions by evaluating the vascular involvement of the tumor. If the pathology report was insufficiently detailed to determine $\mathrm{T}$ category, preoperative imaging was also used.

\section{Statistical analysis}

Statistical analyses were performed using IBM SPSS Statistics for Windows ver. 25.0 (IBM Corp., Armonk, NY). Categorical data are reported as counts and percentages. Nominal data were compared using chi-square tests and continu-

Table 1. American Joint Committee on Cancer (AJCC) staging system

\begin{tabular}{llll} 
& AJCC, 7th edition & & AJCC, 8th edition \\
I & T1 N0 M0 & I & T1 N0 M0 \\
II & T2a-b N0 M0 & II & T2a-b N0 M0 \\
IIIA & T3 N0 M0 & IIIA & T3 N0 M0 \\
IIIB & T1-3 N1 M0 & IIIB & T4 N0 M0 \\
& & IIIC & AnyT N1 M0 \\
IVA & T4 AnyN M0 & IVA & AnyT N2 M0 \\
IVB & AnyT N2 M0 & IVB & AnyT AnyN M1 \\
& AnyT AnyN M1 & & \\
\hline
\end{tabular}


Table 2. Patient demographics and perioperative outcomes

\begin{tabular}{|c|c|}
\hline Characteristic & Value $(n=348)$ \\
\hline Age (yr) & $63.8 \pm 8.9$ \\
\hline$\geq 65 \mathrm{yr}$ & $173(49.7)$ \\
\hline Male sex & $232(66.7)$ \\
\hline Diabetes & $64(18.4)$ \\
\hline Total bilirubin (mg/dL) & $1.8 \pm 1.4$ \\
\hline Albumin $(g / d L)$ & $3.3 \pm 0.5$ \\
\hline CA19-9 (U/mL) & $536.1 \pm 2,306$ \\
\hline Preoperative portal vein embolization & $8(2.3)$ \\
\hline \multicolumn{2}{|l|}{ Liver resection type } \\
\hline Right hepatectomy & $224(64.4)$ \\
\hline Right trisectionectomy & $15(4.3)$ \\
\hline Left hepatectomy & $86(24.7)$ \\
\hline Left trisectionectomy & $20(5.7)$ \\
\hline Central bisectionectomy & $3(0.9)$ \\
\hline Portal vein resection and reconstruction & $54(15.5)$ \\
\hline Intraoperative transfusion & $127(36.5)$ \\
\hline \multicolumn{2}{|l|}{ Bismuth type } \\
\hline I & $12(3.4)$ \\
\hline II & $52(14.9)$ \\
\hline IIIa & $161(46.3)$ \\
\hline $\mathrm{IIIlb}$ & $61(17.5)$ \\
\hline IV & $62(17.8)$ \\
\hline \multicolumn{2}{|l|}{ Tumor size (cm) } \\
\hline$\geq 3$ & $176(50.7)$ \\
\hline$<3$ & $171(49.3)$ \\
\hline \multicolumn{2}{|l|}{ Histologic differentiation } \\
\hline WD & $68(19.5)$ \\
\hline $\mathrm{MD}$ & $227(65.2)$ \\
\hline PD & 44 (12.6) \\
\hline Others & $9(2.6)$ \\
\hline Lymph node involvement & $135(38.8)$ \\
\hline Lymphovascular invasion & $169(48.6)$ \\
\hline Perineural invasion & $288(82.8)$ \\
\hline $\mathrm{R} 0$ resection & $216(62.1)$ \\
\hline PHLF (ISGLS B or C) & $40(11.5)$ \\
\hline Hospital stay (day) & $21.8 \pm 14.1$ \\
\hline 90-Day mortality & $10(2.9)$ \\
\hline Adjuvant chemotherapy & $134(38.5)$ \\
\hline Overall survival (mo) & $34.2 \pm 26.0$ \\
\hline Disease-free survival (mo) & $25.2 \pm 25.1$ \\
\hline
\end{tabular}

Values are presented as mean \pm standard deviation or number $(\%)$. CA19-9, carbohydrate antigen 19-9; WD, well differentiated; $\mathrm{MD}$, moderate differentiated; $\mathrm{PD}$, poorly differentiated; PHLF, posthepatectomy liver failure; ISGLS, International Study Group of Liver Surgery. ous variables were compared using Student $t$ tests. Estimates of OS were calculated using the Kaplan-Meier method and compared with the log-rank test. Cox proportional hazard models were used to evaluate prognostic factors associated with OS. The concordance index (C-index) was used to compare the power of risk prediction between the 7th and 8th AJCC staging systems [4]. The concordance index is a measure of discrimination used to assess whether a staging system can distinguish between two patients at different stages. A concordance index of 0.5 means that the predictive value is no better than random chance and 1 implicates a perfect prediction. $\mathrm{p}$-values less than 0.05 were considered statistically significant.

\section{Ethical statement}

This study was approved by our institutional review board (AMC2018-1421) with a waiver of the informed consent. Clinicopathologic data including information on survival and disease recurrence was obtained from electronic medical records and retrospectively reviewed.

\section{Results}

\section{Clinicopathologic characteristics}

During the study period, a total of 348 patients with PHCC underwent surgical resection with major hepatectomy. Table 2 shows the demographics and perioperative outcomes of all the included patients. A total of 232 men and 116 women with an overall mean age of 63.8 years were included. Right $(\mathrm{n}=224,64.4 \%)$ and left $(\mathrm{n}=86,24.7 \%)$ hepatectomy were most frequently performed. Combined portal vein resection and reconstruction was performed in 54 patients $(15.5 \%)$.

According to the Bismuth classification, type IIIa ( $\mathrm{n}=161$, $46.3 \%$ ) was most common, followed by type IV $(n=62,17.8 \%)$ and type IIIb $(\mathrm{n}=61,17.5 \%)$. R0 resection rate of Bismuth type IV tumor was $53.2 \%$ (33 out of 62 patients), which was slightly lower than the overall R0 rate of $62.1 \%$. In the Kaplan-Meier survival analysis, there was no significant poor survival rate in Bismuth type IV; rather, a difference between type II and III ( $\mathrm{p}=0.046)$ was observed. In pathologic data, LN involvement was found in 135 patients (38.8\%).

In clinical data, the 90 -day mortality rate was $2.9 \%$ and 134 patients $(38.5 \%)$ received adjuvant chemotherapy after surgery. Mean OS and disease-free survival were 34.2 and 25.2 months, respectively. 
Table 3. Cross-tabulation of the 7th and 8th editions of the AJCC staging system: T-, N-, and AJCC stage

\begin{tabular}{|c|c|c|c|c|c|c|c|}
\hline \multirow{2}{*}{ T stage } & \multicolumn{6}{|c|}{ 7th edition } & \multirow{2}{*}{ Total } \\
\hline & \multicolumn{3}{|c|}{$\mathrm{T} 2$} & \multicolumn{2}{|c|}{ T3 } & $\mathrm{T} 4$ & \\
\hline \multicolumn{8}{|l|}{ 8th edition } \\
\hline $\mathrm{T} 1$ & 39 & \multicolumn{2}{|c|}{-} & \multicolumn{2}{|l|}{-} & 5 & 44 (12.6) \\
\hline $\mathrm{T} 2$ & - & \multicolumn{2}{|c|}{181} & \multicolumn{2}{|l|}{-} & 23 & $204(58.6)$ \\
\hline T3 & - & \multicolumn{2}{|c|}{-} & \multicolumn{2}{|c|}{44} & 22 & 66 (18.9) \\
\hline $\mathrm{T} 4$ & - & \multicolumn{2}{|c|}{-} & \multicolumn{2}{|c|}{-} & 34 & 35 (10.1) \\
\hline Total & 39 (11.2) & \multicolumn{2}{|c|}{$181(52.0)$} & \multicolumn{2}{|c|}{44 (12.6) } & $84(24.1)$ & 348 \\
\hline \multirow{2}{*}{ N stage } & \multicolumn{6}{|c|}{ 7th edition } & \multirow{2}{*}{ Total } \\
\hline & No & & & & & N2 & \\
\hline \multicolumn{8}{|l|}{ 8th edition } \\
\hline N0 & 212 & \multicolumn{4}{|c|}{-} & - & $212(60.9)$ \\
\hline N1 & - & \multicolumn{4}{|c|}{100} & 2 & $102(29.3)$ \\
\hline N2 & - & \multicolumn{4}{|c|}{30} & 4 & $34(9.8)$ \\
\hline Total & $212(60.9)$ & \multicolumn{4}{|c|}{$130(37.3)$} & $6(1.7)$ & 348 \\
\hline \multirow{2}{*}{ AJCC stage } & \multicolumn{6}{|c|}{ 7th edition } & \multirow{2}{*}{ Total } \\
\hline & I & II & IIIA & IIIB & IVA & IVB & \\
\hline \multicolumn{8}{|l|}{ 8th edition } \\
\hline I & 31 & - & - & - & 5 & - & $36(10.3)$ \\
\hline II & - & 111 & - & - & 14 & - & 125 (35.9) \\
\hline IIIA & - & - & 23 & - & 11 & - & $34(9.8)$ \\
\hline IIIB & - & - & - & - & 17 & - & $17(4.9)$ \\
\hline IIIC & - & - & - & 71 & 25 & 1 & 97 (27.9) \\
\hline IVA & - & - & 1 & 20 & 7 & 3 & $31(8.9)$ \\
\hline IVB & - & - & - & 2 & - & 6 & $8(2.3)$ \\
\hline Total & $31(8.9)$ & 111 (31.9) & $24(6.9)$ & $93(26.7)$ & 79 (22.7) & $10(2.9)$ & 348 \\
\hline
\end{tabular}

Values are presented as number $(\%)$. AJCC, American Joint Committee on Cancer.

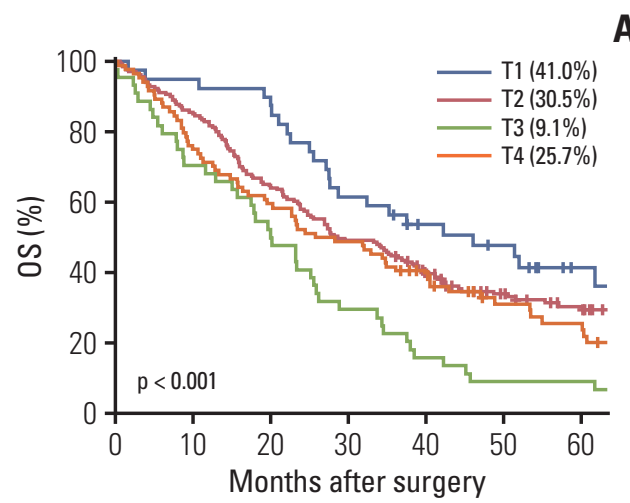

A

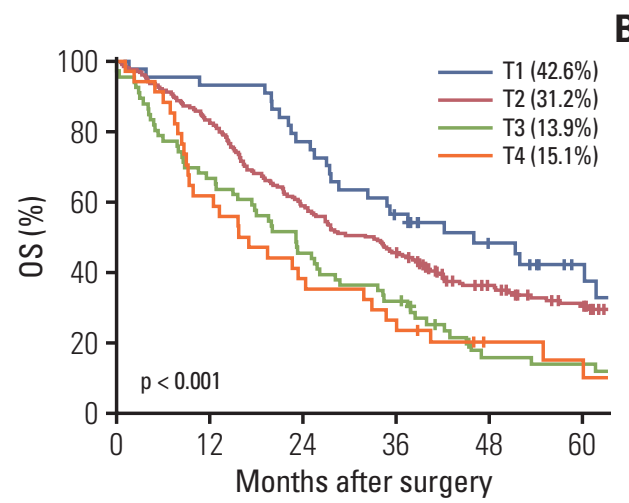

Fig. 1. Five-year overall survival (OS) rate. (A) The 7th American Joint Committee on Cancer (AJCC) T classification, T1 vs. T2 ( $p=0.262)$, T2 vs. T3 ( $p<0.001)$, T3 vs. T4 ( $p=0.013)$. (B) The 8th AJCC T classification., T1 vs. T2 ( $p=0.260), T 2$ vs. T3 $(\mathrm{p}=0.001)$, T3 vs. T4 $(\mathrm{p}=0.996)$. 
A
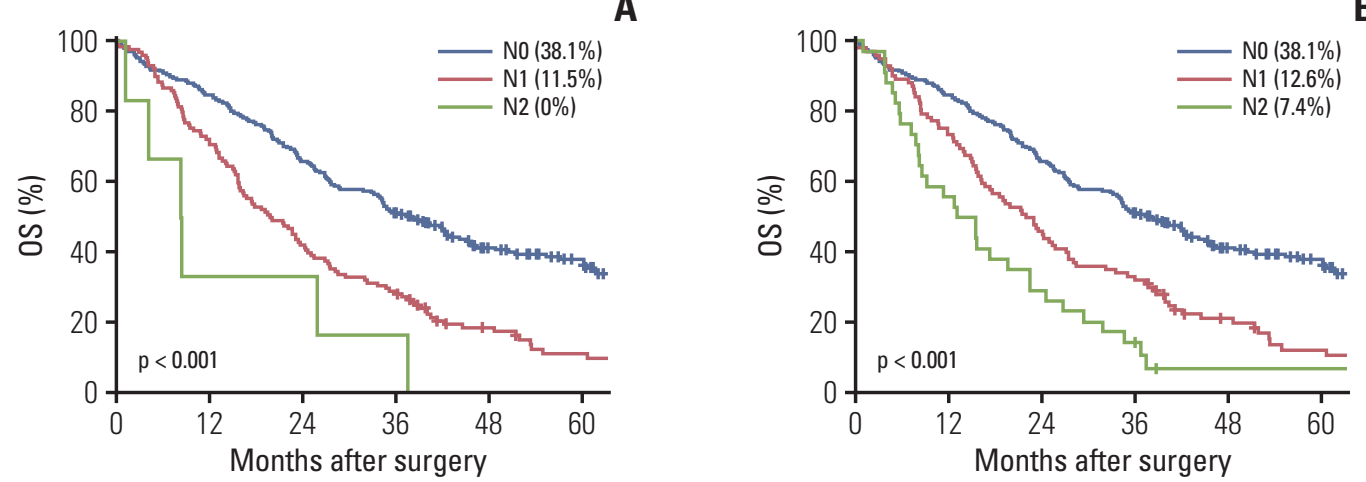

Fig. 2. Five-year overall survival (OS) rate. (A) The 7th American Joint Committee on Cancer (AJCC) N classification, N0 vs. N1 ( $\mathrm{p}<0.001)$, N1 vs. N2 ( $\mathrm{p}=0.063)$. (B) The 8th AJCC N classification, N0 vs. N1 ( $<<0.001)$, N1 vs. N2 ( $\mathrm{p}=0.016)$.

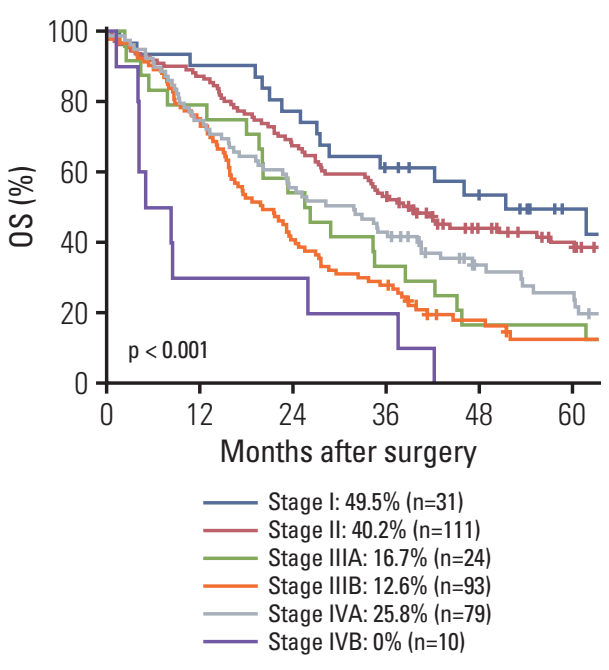

A

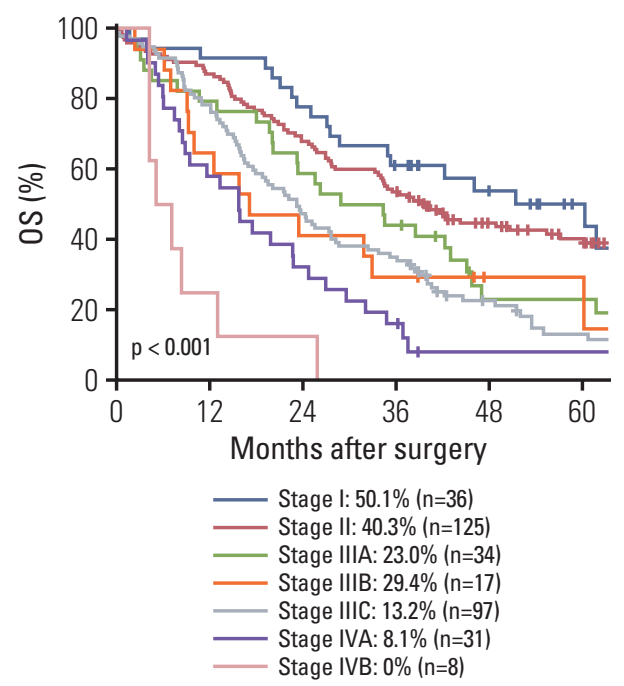

Fig. 3. Five-year overall survival (OS) rate according to the 7th American Joint Committee on Cancer (AJCC) stage, I vs. II ( $\mathrm{p}=0.644)$, II vs. IIIA ( $\mathrm{p}=0.007)$, IIIA vs. IIIB ( $\mathrm{p}=0.723)$, IIIB vs. IVA ( $\mathrm{p}=0.047)$, IVA vs. IVB ( $\mathrm{p}=0.001)$ (A) and the 8th AJCC

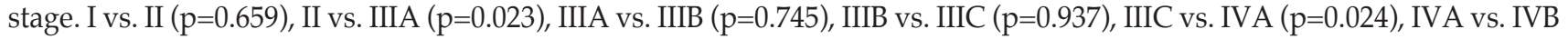
$(\mathrm{p}=0.013)(B)$.

\section{Stage transitions}

Table 3 is a cross-tabulation presenting transitions for T-, $\mathrm{N}-$, and AJCC stages for the 7th and 8th staging editions. A total of 170 patients $(48.9 \%)$ were reclassified considering substages (e.g., stage IIIA and IIIB). Staging according to the 8th edition downstaged 76 patients $(21.8 \%)$ and upstaged 94 patients $(27 \%)$ in comparison with the 7 th edition.

\section{Survival across stages}

Figs. 1, 2, and 3 present the Kaplan-Meier curves for OS of the $\mathrm{T}-, \mathrm{N}-$, and overall stage groups of the 7th and 8th editions. Findings of the log-rank tests for both staging systems were statistically significant $(\mathrm{p}<0.001)$.

\section{Validation of $\mathrm{T}$ classification}

The numbers of patients with $\mathrm{T} 1, \mathrm{~T} 2, \mathrm{~T} 3$, and $\mathrm{T} 4$ disease, according to the 7th AJCC T staging system, were 39 (11.2\%), 
Table 4. Comparison of prognostic predictability between the 8th and 7th T-, $\mathrm{N}-$, and AJCC stages

\begin{tabular}{|c|c|c|c|}
\hline & C-index & $95 \% \mathrm{CI}$ & p-value \\
\hline 8th T classification & 0.581 & 0.550 to 0.613 & \\
\hline 7th T classification & 0.553 & 0.519 to 0.588 & \\
\hline Difference & 0.028 & 0.008 to 0.048 & 0.005 \\
\hline 8th $\mathrm{N}$ classification & 0.593 & 0.562 to 0.625 & \\
\hline 7th $\mathrm{N}$ classification & 0.587 & 0.556 to 0.619 & \\
\hline Difference & 0.006 & -0.001 to 0.013 & 0.115 \\
\hline 8th AJCC stage & 0.621 & 0.587 to 0.655 & \\
\hline 7th AJCC stage & 0.582 & 0.545 to 0.619 & \\
\hline Difference & 0.039 & 0.012 to 0.067 & 0.005 \\
\hline
\end{tabular}

AJCC, American Joint Committee on Cancer; CI, confidence interval.

$181(52.0 \%), 44(12.6 \%)$, and $84(24.1 \%)$, respectively. The numbers of patients with T1, T2, T3, and T4, according to the 8th AJCC T staging system, were $44(12.6 \%), 204(58.6 \%), 66$ $(18.9 \%)$, and $35(10.1 \%)$, respectively.

In terms of stage migration, all patients of the 7th T1, T2, and $\mathrm{T} 3$ categories remained in the same stage as in the 8th T staging. However, 50 patients $(14.4 \%)$ in the 7 th T4 category moved to other categories in the 8th edition.

Fig. $1 \mathrm{~A}$ and $\mathrm{B}$ show the 5-year OS rate according to the 7th and 8th AJCC T classification, respectively. A log-rank test revealed that 5-year OS rates showed significant differences between the 7th edition T2 and T3 ( $<<0.001)$ and T3 and T4 $(\mathrm{p}=0.013)$. While the survival rate of T4 decreased in the 8 th edition, significant differences were only seen between T2 and T3 $(\mathrm{p}=0.001)$.

The C-indexes of the 7th and 8th $\mathrm{T}$ classifications were 0.553 and 0.581, respectively (Table 4). Differences between both editions were statistically significant $(p=0.005)$, which meant that the difference in prognostic predictability improved in the 8 th $\mathrm{T}$ classification.

\section{Validation of $\mathbf{N}$ classification}

The numbers of patients with N0, N1, and N2 according to the 8th edition were $212(60.9 \%), 102(29.3 \%)$, and 34 $(9.8 \%)$, respectively. Thirty-two patients moved to other stages in the 8th edition, with most of them migrating from $\mathrm{N} 1$ to N2. In terms of survival, both editions showed significant differences in survival between each stage, except between N1 and N2 ( $p=0.063)$ in 7th edition.

The C-indexes of the 7 th and 8th $\mathrm{N}$ classifications were 0.587 and 0.593 , respectively (Table 4 ). However, the $95 \%$ confidence interval (CI) of the difference was -0.001 to 0.013 , which means that the difference in prognostic predictability between both $\mathrm{N}$ classifications was not statistically significant $(\mathrm{p}=0.115)$.

\section{Validation of overall disease stage}

A total of 170 patients (48.8\%) changed stages from 7 th to the 8 th edition of the AJCC overall staging system.

Regarding survival, IVA corresponding to T4 showed a relatively good survival rate (5-year survival rate, $25.8 \%$ ) in the 7 th edition, and IIIB corresponding to T4 in the 8th edition improved (5-year survival rate, 29.4\%), which appears to have improved in terms of survival discrimination.

The calculated C-indexes of the 7th and 8th AJCC stages were 0.582 and 0.621 , respectively. The difference in C-index between the two editions was 0.039 and the $95 \% \mathrm{CI}$ was 0.012 to $0.067(\mathrm{p}=0.005)$.

\section{Prognostic factor}

Several clinicopathologic factors were analyzed to assess their association with OS (Table 5). Univariate analysis identified the following predictors of survival: posthepatectomy liver failure (PHLF; International Study Group of Liver Surgery grade $B$ or $C, p<0.001$ ), histologic differentiation (poorly differentiated, moderate differentiated vs. well differentiated, $\mathrm{p}=0.005)$, $\mathrm{T}$ category (T3, $\mathrm{p}=0.001$ and $\mathrm{T} 4, \mathrm{p}=0.004), \mathrm{N}$ category (N1 and N2, p < 0.001), distant metastasis ( $<<$ $0.001)$, intraoperative transfusion $(\mathrm{p}=0.001)$, resection margin (R1 vs. R0, $\mathrm{p}<0.001)$, and lymphovascular invasion ( $\mathrm{p}<$ 0.001).

The following prognostic factors also showed independent association with survival in the multivariate analysis: PHLF (hazard ratio [HR], 3.47; $\mathrm{p}<0.001$ ), T category (T3 vs. T1; HR, 1.69; $\mathrm{p}=0.042$ and T4 vs. T1; HR, 2.61; $\mathrm{p}=0.004), \mathrm{N}$ category (N1 vs. N0; HR, 1.69; $\mathrm{p}<0.001$ and N2 vs. N0; HR, 2.41; $\mathrm{p}<$ $0.001)$, distant metastasis (HR, 3.90; $\mathrm{p}<0.001)$, histologic differentiation ( $\mathrm{HR}, 1.75 ; \mathrm{p}=0.002)$, intraoperative transfusion $(\mathrm{HR}, 1.58 ; \mathrm{p}=0.001)$, and resection margin status (HR, 1.32; $\mathrm{p}=0.043$ ). 
Table 5. Univariate and multivariate analyses of survival in 348 patients

\begin{tabular}{|c|c|c|c|c|}
\hline & \multicolumn{2}{|c|}{ Univariate analysis } & \multicolumn{2}{|c|}{ Multivariate analysis } \\
\hline & $\mathrm{HR}(95 \% \mathrm{CI})$ & p-value & HR $(95 \%$ CI $)$ & p-value \\
\hline Age ( $\geq 65$ yr vs. $<65$ yr) & $1.142(0.898-1.452)$ & 0.281 & $1.291(0.989-1.683)$ & 0.060 \\
\hline Sex (male vs. female) & $1.025(0.795-1.321)$ & 0.851 & - & - \\
\hline Diabetes (yes vs. no) & $0.932(0.678-1.280)$ & 0.662 & - & - \\
\hline CA19-9 ( $n=336)(\geq 37$ vs. $<38)$ & $1.139(0.878-1.478)$ & 0.326 & - & - \\
\hline Hepatectomy type & & 0.227 & - & - \\
\hline CBS & Ref & Ref & & \\
\hline $\mathrm{RH}$ & $0.385(0.122-1.211)$ & 0.103 & - & - \\
\hline RTS & $0.429(0.120-1.528)$ & 0.191 & - & - \\
\hline LH & $0.430(0.096-0.989)$ & 0.048 & - & - \\
\hline LTS & $0.403(0.116-1.399)$ & 0.152 & - & - \\
\hline Portal vein resection & $1.365(0.990-1.882)$ & 0.057 & $0.543(0.344-0.857)$ & 0.009 \\
\hline Resection margin status (R1 vs. R0) & $1.572(1.232-2.006)$ & $<0.001$ & $1.322(1.008-1.732)$ & 0.043 \\
\hline Intraoperative transfusion & $1.522(1.191-1.945)$ & 0.001 & $1.582(1.208-2.071)$ & 0.001 \\
\hline Bismuth type & & 0.219 & & - \\
\hline I & Ref & Ref & Ref & Ref \\
\hline II & $1.056(0.492-2.264)$ & 0.890 & $0.698(0.327-1.492)$ & 0.353 \\
\hline III & $1.553(0.758-3.183)$ & 0.229 & $1.016(0.511-2.022)$ & 0.964 \\
\hline IV & $1.258(0.593-2.673)$ & 0.550 & $0.578(0.276-1.214)$ & 0.148 \\
\hline PHLF (ISGLS B or C) & $2.516(1.766-3.585)$ & $<0.001$ & $3.472(2.369-5.089)$ & $<0.001$ \\
\hline Histologic differentiation (PD, MD vs. WD) & $1.567(1.147-2.141)$ & 0.005 & $1.749(1.232-2.484)$ & 0.002 \\
\hline AJCC T stage (8th) & $1.712(1.327-2.210)$ & $<0.001$ & & $<0.001$ \\
\hline $\mathrm{T} 1$ & Ref & Ref & Ref & Ref \\
\hline $\mathrm{T} 2$ & $1.245(0.840-1.845)$ & 0.275 & $0.845(0.555-1.287)$ & 0.433 \\
\hline T3 & $2.098(1.352-3.254)$ & 0.001 & $1.692(1.020-2.805)$ & 0.042 \\
\hline $\mathrm{T} 4$ & $2.121(1.271-3.549)$ & 0.004 & $2.614(1.360-5.025)$ & 0.004 \\
\hline \multicolumn{5}{|l|}{ AJCC N stage (8th) } \\
\hline N0 & Ref & Ref & Ref & Ref \\
\hline N1 & $1.740(1.332-2.273)$ & $<0.001$ & $1.689(1.260-2.263)$ & $<0.001$ \\
\hline N2 & $2.840(1.915-4.212)$ & $<0.001$ & $2.405(1.567-3.691)$ & $<0.001$ \\
\hline Distant metastasis & $5.712(2.780-11.736)$ & $<0.001$ & $3.904(1.737-8.775)$ & $<0.001$ \\
\hline Tumor size $(\geq 3 \mathrm{~cm}$ vs. $<3 \mathrm{~cm})$ & $1.231(0.968-1.567)$ & 0.090 & - & - \\
\hline Lymphovascular invasion & $1.656(1.299-2.111)$ & $<0.001$ & - & - \\
\hline Perineural invasion & $1.389(0.995-1.938)$ & 0.053 & - & - \\
\hline
\end{tabular}

$\mathrm{HR}$, hazard ratio; CI, confidence interval; CA 19-9, cancer antigen 19-9; CBS, central bisectionectomy; $\mathrm{RH}$, right hepatectomy; RTS, right trisectionectomy; LH, left hepatectomy; LTS, left trisectionectomy; PHLF, posthepatectomy liver failure; ISGLS, International Study Group of Liver Surgery; PD, poorly differentiated; $\mathrm{MD}$, moderate differentiated; WD, well differentiated; AJCC, American Joint Committee on Cancer.

\section{Discussion}

This study compared the prognostic value of 7 th and 8 th editions of the AJCC staging system in patients with resected PHCC. As previously mentioned, the major changes from the 7th to the 8th edition were that Bismuth type IV was removed from the $\mathrm{T} 4$ category and the $\mathrm{N}$ category was reclassified on the basis of the number of positive LNs. In addition, based on the data by Ebata et al. [2] that T4 disease (pT4N0M0) had better survival than patients with regional node-positive disease (pN1M0) and LN metastasis was a strong prognostic factor, in the overall stage group, T4 was downstaged from stage IVA to IIIB, N1 category was changed from stage IIIB to IIIC, and N2 category was classified as stage IVA.

These changes are based on several Japanese reports that showed favorable results after extensive operation for PHCC [5-11]. The number of Bismuth type IV $(n=485)$ and vascular 
resections ( $\mathrm{n}=440)$ in 1,000 resections in eight highly chosen Japanese centers were much larger than those in Western studies [12-14]. Ebata et al. [2] insisted that poorer survival of Bismuth type IV was associated with higher incidence of pM1, pN1, and R1-2; hence, Bismuth tumor type merely describes tumor location rather than resectability or survival prediction. These findings supported the resection of Bismuth type IV tumors and elimination of Bismuth type IV tumors from the definition of Union for International Cancer Control (UICC) T4 [2].

As a result of changing the staging system, the median OS of T4 decreased from 25.7 months in the 7th to 15.7 months in the 8th, and the 5-year OS of T4 also decreased from $25.7 \%$ in the 7 th to $15.1 \%$ in the 8 th. These results show that classification according to survival has been improved in terms of $\mathrm{T}$ category. In overall stage in the 8 th edition, 5 -year survival rate of IIIB $(29.4 \%)$ was slightly better than for IIIC (13.2\%) but this difference was not statistically significant $(\mathrm{p}=0.937)$.

In terms of change in the T classification, the removal of Bismuth type IV from the T4 category seems reasonable even without considering the resection margin and LN status, because the survival rate of Bismuth type IV was not worse compared to the other types. Five-year survival rate of Bismuth type I, II, III, and IV tumors were $33.3 \%, 38.1 \%, 24.0 \%$, and $29.8 \%$, respectively (type I vs. II, $\mathrm{p}=0.912$; II vs. III, $\mathrm{p}=$ 0.046; III vs. IV, $\mathrm{p}=0.212$; I vs. III, $\mathrm{p}=0.335$; II vs. IV, $\mathrm{p}=0.368$ ). Fifty patients whose $\mathrm{T}$ stage had been changed show improved prognostic predictability in T category, which could be confirmed by the $\mathrm{C}$-index $(\mathrm{p}=0.005)$.

Another issue regarding the $\mathrm{T}$ classification is whether the subdivision of T2 is necessary. Survival between pT2b tumors (liver invasion) and pT2a (invasion beyond the ductal wall) was not statistically different (5-year survival rate of $\mathrm{T} 2 \mathrm{a} 33.5 \%$, T2b 28.7\%, $\mathrm{p}=0.168$ ) which is consistent with previous Japanese data [2]. Further studies would be required to determine the impact of liver invasion on survival.

In terms of $\mathrm{N}$ classification, multivariate analysis showed that the number of positive metastatic LNs (MLNs) was strongly associated with prognosis (1-3 MLNs vs. N0: HR, $1.69 ; 4$ or more MLNs vs. N0: HR, 2.41). These results support the change of the $\mathrm{N}$ category to a classification based on the number of MLNs. However, the difference in prognostic predictability between both $\mathrm{N}$ editions showed no significance as calculated by $\mathrm{C}$-index $(\mathrm{p}=0.115)$.

There have been some studies advocating different methods of stratifying LN involvement to better predict the longterm outcome for other cancers including pancreatic, gastric, and biliary cancer. Some studies suggested that the LN ratio (LNR), which is the ratio of the number of MLNs and the total number of LNs retrieved (TLN) is a better prognostic indicator $[15,16]$. In another study, log odds of MLNs (LODDS), which is defined as the natural logarithm of the ratio between the probability of an LN to contain tumor cells and the probability of an LN to be free of tumor cells, was recently suggested as an alternative tool for evaluating LN status [17]. In our data, the area under the receiver operating characteristic curve (AUC) predicting the 5-year OS of LNR (AUC, 0.671) was greater than LODDS (AUC, 0.598) and AJCC 8th $\mathrm{N}$ category (AUC, 0.666).

Some authors recommended histologic examination for more than a certain number of LNs; however, the minimum required number of LNs is still controversial. The median number of LNs harvested in this cohort was 9 which is similar to that in other Western studies $[18,19]$ and the incidence of LN metastasis was $38.8 \%$ ( $\mathrm{n}=135)$. The difference in survival was not significant in most of the TLN cutoff values, but we confirmed a clear tendency that the greater the TLN cutoff value, the smaller the difference in survival. This suggests that inadequate assessment of LNs could lead to understaging in patients with a low TLN. There have been some reports on the minimum requirements of the histologic examination of regional LNs in PHCC [20-22]. One of them recommended five LNs to obtain adequate LN staging [20] and another study from Western centers suggested that four to seven LNs may be adequate for correct staging [22,23]. In our data, a suitable minimum number of LN has not been calculated because the greatest difference of survival was seen in one TLN. To prevent understaging, we need to remove all soft tissue around the regional LNs and cooperate with pathologists.

In addition, previous studies have provided further insight into $\mathrm{T}$ staging by pointing out that the extrahepatic bile duct consists of a relatively thin, variable, and complex fibromuscular layer [24], which makes it difficult for a pathologist to discriminate each layer, in contrast to other gastrointestinal organs with concentric and well-discriminated layers [25]. Some reports have demonstrated the prognostic value of tumor depth in PHCC and proposed a new T system with tumor depth incorporating all extrahepatic bile duct cancers $[26,27]$. These studies indicate the need for further pathologic research to obtain a conclusion.

There are two previous European reports evaluating prognostic accuracy of AJCC 8th staging system of perihilar cholangiocarcinoma. One from Italy by Ruzzenente et al. [28] included 214 patients who underwent surgery. They concluded 8th edition was slightly improved compared to 7 th edition (C-index: 0.619, 7th; 0.624, 8th). The other from the Netherlands by Gaspersz et al. [29] analyzed total of 248 patients including 45 underwent curative resection and 243 with unresectable patients. Their conclusion was both editions showed comparable prognostic accuracy (C-index: $0.57,7$ th; $0.58,8$ th).

More efforts and research are needed in many ways to improve outcome of PHCC patients. In this study, PHLF, 
TNM category, tumor differentiation, intraoperative transfusion and resection margin were found to be the prognostic factor. Yoo et al. [30] reported clear resection margin was the most important prognostic factors for survival but aggressive resection should be carefully performed considering the condition of each patients. Further research such as subgroup analysis for each prognostic factor will also be needed.

The present study has several limitations. Firstly, this study is a single-center, retrospective study, similar to many other studies on PHCC. Secondly, there could have been selection bias given that only patients undergoing major hepatectomy with resectable disease were included in this study. However, at the same time, it was an advantage that pathological confirmation of the TNM stage was available for all patients. Thirdly, the number of patients with severe staging, such as T4 or N2, was too small to calculate survival outcomes and derive statistical power. Lastly, the number of examined LNs in the final pathology varied somewhat from 1 to 52 (mean, 10.22; median number, 9.0 0 7.67). There were 52 patients with less than three LNs examined, the possibility of understaging cannot be ruled out and further analysis will be needed.

In conclusion, the prognostic predictability of 8th AJCC staging for PHCC seems to have slightly improved, with statistical significance, over the previous edition, but the overall performance is still unsatisfactory (C-index $<0.7$ ). Even if previous studies have shown similar results $[28,29]$, the assessment of substantial changes in the 8th AJCC staging for PHCC remains controversial. If further studies continue to show improved results from based on the new system, we could produce a definitive conclusion on this matter, and the present study may serve as a basis for these.

\section{Conflicts of Interest}

Conflict of interest relevant to this article was not reported.

\section{Acknowledgments}

This study was presented at the 7th biennial congress of the Asian-Pacific Hepato-Pancreato-Biliary Association (A-PHPBA), Seoul, Republic of Korea, Sep. 2019.

\section{References}

1. Ebata T, Kamiya J, Nishio H, Nagasaka T, Nimura Y, Nagino $\mathrm{M}$. The concept of perihilar cholangiocarcinoma is valid. Br J Surg. 2009;96:926-34.

2. Ebata T, Kosuge T, Hirano S, Unno M, Yamamoto M, Miyazaki $\mathrm{M}$, et al. Proposal to modify the International Union Against Cancer staging system for perihilar cholangiocarcinomas. Br J Surg. 2014;101:79-88.

3. Chun YS, Pawlik TM, Vauthey JN. 8th edition of the AJCC cancer staging manual: pancreas and hepatobiliary cancers. Ann Surg Oncol. 2018;25:845-7.

4. Steyerberg EW, Vickers AJ, Cook NR, Gerds T, Gonen M, Obuchowski N, et al. Assessing the performance of prediction models: a framework for traditional and novel measures. Epidemiology. 2010;21:128-38.

5. Ebata T, Nagino M, Kamiya J, Uesaka K, Nagasaka T, Nimura Y. Hepatectomy with portal vein resection for hilar cholangiocarcinoma: audit of 52 consecutive cases. Ann Surg. 2003;238: 720-7.

6. Ebata T, Yokoyama Y, Igami T, Sugawara G, Takahashi Y, Nimura $Y$, et al. Hepatopancreatoduodenectomy for cholangiocarcinoma: a single-center review of 85 consecutive patients. Ann Surg. 2012;256:297-305.

7. Esaki M, Shimada K, Nara S, Kishi Y, Sakamoto Y, Kosuge T, et al. Left hepatic trisectionectomy for advanced perihilar cholangiocarcinoma. Br J Surg. 2013;100:801-7.

8. Miyazaki M, Kato A, Ito H, Kimura F, Shimizu H, Ohtsuka M, et al. Combined vascular resection in operative resection for hilar cholangiocarcinoma: does it work or not? Surgery. 2007; 141:581-8.

9. Nagino M, Kamiya J, Arai T, Nishio H, Ebata T, Nimura Y. "Anatomic" right hepatic trisectionectomy (extended right hepatectomy) with caudate lobectomy for hilar cholangiocarcinoma. Ann Surg. 2006;243:28-32.

10. Nagino M, Nimura $Y$, Nishio H, Ebata T, Igami T, Matsushita $\mathrm{M}$, et al. Hepatectomy with simultaneous resection of the portal vein and hepatic artery for advanced perihilar cholangiocarcinoma: an audit of 50 consecutive cases. Ann Surg. 2010; 252:115-23.

11. Seyama Y, Kubota K, Sano K, Noie T, Takayama T, Kosuge T, et al. Long-term outcome of extended hemihepatectomy for hilar bile duct cancer with no mortality and high survival rate. Ann Surg. 2003;238:73-83.

12. de Jong MC, Marques H, Clary BM, Bauer TW, Marsh JW, Ribero D, et al. The impact of portal vein resection on outcomes for hilar cholangiocarcinoma: a multi-institutional analysis of 305 cases. Cancer. 2012;118:4737-47.

13. Nuzzo G, Giuliante F, Ardito F, Giovannini I, Aldrighetti L, Belli $G$, et al. Improvement in perioperative and long-term outcome after surgical treatment of hilar cholangiocarcinoma: results of an Italian multicenter analysis of 440 patients. Arch Surg. 2012;147:26-34.

14. Regimbeau JM, Fuks D, Le Treut YP, Bachellier P, Belghiti J, Boudjema K, et al. Surgery for hilar cholangiocarcinoma: a multi-institutional update on practice and outcome by the 
AFC-HC study group. J Gastrointest Surg. 2011;15:480-8.

15. Guglielmi A, Ruzzenente A, Campagnaro T, Valdegamberi A, Bagante F, Bertuzzo F, et al. Patterns and prognostic significance of lymph node dissection for surgical treatment of perihilar and intrahepatic cholangiocarcinoma. J Gastrointest Surg. 2013;17:1917-28.

16. Sakata J, Wakai T, Matsuda Y, Ohashi T, Hirose Y, Ichikawa $\mathrm{H}$, et al. Comparison of number versus ratio of positive lymph nodes in the assessment of lymph node status in extrahepatic cholangiocarcinoma. Ann Surg Oncol. 2016;23:225-34.

17. Conci S, Ruzzenente A, Sandri M, Bertuzzo F, Campagnaro T, Bagante $\mathrm{F}$, et al. What is the most accurate lymph node staging method for perihilar cholangiocarcinoma? Comparison of UICC/AJCC pN stage, number of metastatic lymph nodes, lymph node ratio, and log odds of metastatic lymph nodes. Eur J Surg Oncol. 2017;43:743-50.

18. Bagante F, Tran T, Spolverato G, Ruzzenente A, Buttner S, Ethun CG, et al. Perihilar cholangiocarcinoma: number of nodes examined and optimal lymph node prognostic scheme. J Am Coll Surg. 2016;222:750-9.

19. Giuliante F, Ardito F, Guglielmi A, Aldrighetti L, Ferrero A, Calise F, et al. Association of lymph node status with survival in patients after liver resection for hilar cholangiocarcinoma in an Italian multicenter analysis. JAMA Surg. 2016;151:91622.

20. Aoba T, Ebata T, Yokoyama Y, Igami T, Sugawara G, Takahashi $Y$, et al. Assessment of nodal status for perihilar cholangiocarcinoma: location, number, or ratio of involved nodes. Ann Surg. 2013;257:718-25.

21. Guglielmi A, Ruzzenente A, Campagnaro T, Pachera S, Conci S, Valdegamberi A, et al. Prognostic significance of lymph node ratio after resection of peri-hilar cholangiocarcinoma. HPB (Oxford). 2011;13:240-5.

22. Ito K, Ito H, Allen PJ, Gonen M, Klimstra D, D'Angelica MI, et al. Adequate lymph node assessment for extrahepatic bile duct adenocarcinoma. Ann Surg. 2010;251:675-81.

23. Ruys AT, Kate FJ, Busch OR, Engelbrecht MR, Gouma DJ, van Gulik TM. Metastatic lymph nodes in hilar cholangiocarcinoma: does size matter? HPB (Oxford). 2011;13:881-6.

24. Hong SM, Kang GH, Lee HY, Ro JY. Smooth muscle distribution in the extrahepatic bile duct: histologic and immunohistochemical studies of 122 cases. Am J Surg Pathol. 2000;24: 660-7.

25. Kim JH, Bae JM, Oh HJ, Lee HS, Kang GH. Pathologic factors associated with prognosis after adjuvant chemotherapy in stage II/ III microsatellite-unstable colorectal cancers. J Pathol Transl Med. 2015;49:118-28.

26. de Jong MC, Hong SM, Augustine MM, Goggins MG, Wolfgang CL, Hirose K, et al. Hilar cholangiocarcinoma: tumor depth as a predictor of outcome. Arch Surg. 2011;146:697-703.

27. Shinohara K, Ebata T, Shimoyama Y, Nakaguro M, Mizuno T, Matsuo K, et al. Proposal for a new classification for perihilar cholangiocarcinoma based on tumour depth. Br J Surg. 2019; 106:427-35.

28. Ruzzenente A, Bagante F, Ardito F, Campagnaro T, Scoleri I, Conci S, et al. Comparison of the 7th and 8th editions of the American Joint Committee on Cancer Staging Systems for perihilar cholangiocarcinoma. Surgery. 2018;164:244-50.

29. Gaspersz MP, Buettner S, van Vugt JL, de Jonge J, Polak WG, Doukas M, et al. Evaluation of the new American Joint Committee on Cancer Staging Manual 8th Edition for Perihilar Cholangiocarcinoma. J Gastrointest Surg. 2019 Feb 12 [Epub]. https: // doi.org/10.1007/ s11605-019-04127-x.

30. Yoo T, Park SJ, Han SS, Kim SH, Lee SD, Kim TH, et al. Proximal resection margins: more prognostic than distal resection margins in patients undergoing hilar cholangiocarcinoma resection. Cancer Res Treat. 2018;50:1106-13. 